\title{
A systematic review of argumentation techniques for multi-agent systems research
}

\author{
Álvaro Carrera ${ }^{1}$ - Carlos A. Iglesias ${ }^{1}$
}

Published online: 25 July 2015

(C) The Author(s) 2015. This article is published with open access at Springerlink.com

\begin{abstract}
The ability to build arguments that express thoughts is crucial for intelligent interactions among human beings. Thus, argumentation techniques have been applied for years in fields, such as rhetoric or artificial intelligence. More specifically, the agents paradigm fits into the use of these types of techniques because agents shape a society in which they interact to make arrangements or to decide future actions. Those interactions can be modelled using argumentation techniques. Therefore, the application of those techniques in multi-agent systems is an interesting research field. However, no systematic review has been conducted previously, to the best of the authors' knowledge, to provide an overview of argumentation techniques for multi-agent systems. This paper presents a systematic review of argumentation techniques for multi-agent systems research. The period of time that is included in this review is from 1998 to 2014. The objective of this review is to obtain an overview of the existing approaches and to study their impact on research and practice. The research method has been defined to identify relevant studies based on a predefined search strategy, and it is clearly defined to facilitate the reading of this paper. All of the included studies in this review have been analysed from two different points of view: the Application view and the Multi-Agent System view. A comprehensive analysis of the extracted data is provided in the paper, which is based on a set of research questions that are defined. The results of this review reveal suggestions for further research and practice. The argumentation technology is actually in a phase of internal enhancement and exploration. Moreover, the research interest in this topic has increased in the last years. Furthermore, several interesting findings are presented in the paper.
\end{abstract}

Keywords Systematic review $\cdot$ Multi-agent system $\cdot$ Argumentation

\footnotetext{
Álvaro Carrera

a.carrera@dit.upm.es

http://www.gsi.dit.upm.es

Carlos A. Iglesias

cif@dit.upm.es

1 Departamento de Ingeniería de Sistemas Telemáticos, Universidad Politécnica de Madrid, Av. Complutense 30, 28040 Madrid, Spain
} 


\section{Introduction}

Argumentation is a crucial communicative activity in society (Moor and Aakhus 2006); thus, argumentation theory is an interdisciplinary research area (van Eemeren et al. 1996) that has many applications in both theoretical and practical work in fields as computer science and artificial intelligence (Bench-Capon and Dunne 2007).

Multi-Agent Systems is a research area in which argumentation theory has been receiving increasing interest in recent years (Maudet et al. 2007). As a set of agents shape a society, communication among them plays an important role in the system. The techniques used to analyse and design the interaction among these rational agents can use argumentation to facilitate the interaction among them in complex systems. Thus, this work is focused on obtaining conclusions of the current state of the art in order to apply argumentation techniques in a real-life application of a Multi-Agent System (MAS).

The reminder of this paper is structured as follows. Section 2 presents an overview of argumentation theory and some of the most relevant and influential argumentation frameworks. Section 3 shows the research method that has been followed during the review, including the review protocol and the search process. Section 4 presents an overview of the studies included in the review, analysing their data sources or citation status. Section 5 analyses the key aspects defined in the process of data extraction and synthesis. Section 6 offers a discussion of the obtained results. Finally, Sect. 7 presents the conclusions of this work.

\section{Argumentation theory}

Argumentation theory is defined as the interdisciplinary study of the method to obtain conclusions through logical reasoning (van Eemeren et al. 1996). It has been studied in many different fields, such as rhetoric (Wallace 1963; Perelman and Olbrechts-Tyteca 1969), philosophy (Toulmin 2003), law (Feteris 1999) or artificial intelligence (Walton 2009), and includes aspects as debate and negotiation, which are both directed toward achieving valid conclusions and/or agreements. In the literature, we find Dung's work (1995) as one of the most influential approach to argumentation in artificial intelligence field. However, other approaches are widely used in the field, such as Argumentation-based Negotiation (ABN) (Rahwan et al. 2003) or Three-Layer Argumentation Framework (TLAF) (Maio and Silva 2012).

As it is expressed in Dung (1995), "Argumentation constitutes a major component of human intelligence." Thus, the human ability to synthesise ideas in arguments, to understand complex statements, to perform scientific reasoning or, in general, to express their thoughts is a key factor of the intelligent interaction among any being in a society. To transfer this ability to a MAS, a wide range of argumentation frameworks have been applied in many studies (Maudet et al. 2007) in recent years. Many of those frameworks extend a basic and abstract argumentation framework proposed by Dung (1995), which is briefly introduced in Sect. 2.1. Furthermore, other popular argumentation frameworks used in a great variety of studies are described in Sect. 2.2.

\subsection{Basic argumentation framework}

In this section, an introduction to Dung's abstract argumentation framework (1995) is shown to highlight the most important concepts for understanding argumentation theory. This framework is applicable to any field, including philosophy dialogue or sciences debate. 
In this abstract framework, a set of arguments and a set of relations among them are given. Thus, the abstract framework focuses on the definition of the arguments' status. To clarify the concepts of Dung's framework, a set of definitions are shown below.

Definition 2.1 An argumentation framework is a pair $A F=\langle A R, R\rangle$, where $A R$ is a non-empty set of arguments and $R$ is a binary relation on $A R$ called the attack relation $(R \subseteq A R \times A R)$.

Thus, an argumentation framework is defined as a set of arguments and a set of relations among them. An argument can attack another argument to discard it from the reasoning process in order to achieve acceptable statements.

Definition 2.2 Let $a, b \in A R, a$ attacks $b$ (or $b$ is attacked by $a) \Longleftrightarrow(a, b) \in R$.

Definition 2.2 can be extended to sets of arguments. Thus, let $S \subseteq A R, S$ attacks $b$ (or $b$ is attacked by $S) \Longleftrightarrow \exists x \in S \mid(x, b) \in R$.

Definition 2.3 Let $S \subseteq A R \wedge a \in A R, S$ defends $a \Longleftrightarrow S$ attacks $\forall x \in A R \mid(x, a) \in R$.

Thus, a set of arguments can defend a concrete statement and discard (or attack) all of the arguments that attempt to discard that statement.

Definition 2.4 Let $S \subseteq A R ; S$ is conflict-free $\Longleftrightarrow \nexists a, b \in S \mid(a, b) \in R$.

Then, a set of arguments can be considered to be conflict-free when there is no argument that attacks other arguments in the set.

Definition 2.5 An argument $a \in A R$ is acceptable with respect to $S \subseteq A R \Longleftrightarrow \forall b \in$ $A R \mid(b, a) \in R \Rightarrow S$ attacks $b$.

In other words, the acceptable argument with respect to an argument set is composed of all of the arguments that are defended by that set. To know all of the acceptable arguments with respect to a given set of arguments, the characteristic function is defined in Definition 2.6.

Definition 2.6 The characteristic function, denoted by $F_{A F}$ of an argumentation framework $A F=\langle A R, R\rangle$, is defined as follows:

$$
\begin{aligned}
& F_{A F}: 2^{A R} \rightarrow 2^{A R} . \\
& F_{A F}(S)=A \subseteq A R \mid A \text { is acceptable with respect to } S .
\end{aligned}
$$

Once the characteristic function has been defined, there is a set of interesting semantics to reason with the arguments that are shown below.

Definition 2.7 Let $S \subseteq A R \wedge S$ be conflict-free.

$S$ is admissible $\Longleftrightarrow \forall a \in S$ is acceptable with respect to $S$.

Definition 2.8 A preferred extension of an argumentation framework $A F$ is a maximal (with respect to set inclusion) admissible set of $A F$.

Definition 2.9 The grounded extension of an argumentation framework $A F$, denoted by $G E_{A F}$, is the least fixed point of the characteristic function $F_{A F}$, i.e., the best-founded set of arguments. 
Definition 2.10 Let $S \subseteq A R \wedge S$ be conflict-free.

$S$ is a stable extension $\Longleftrightarrow S$ attacks $\forall a \in A R \mid a \notin S$.

With the definitions presented above, the principles of Dung's abstract argumentation framework are summarised. To resolve concrete problems using these principles in a MAS, these concepts are used to set the preferences and strategies of the agents in the system. For exhaustive reading, a set of interesting properties, which are used to simplify the agent reasoning cycle in the argumentation process, are provided by Dung (1995).

\subsection{Extended argumentation frameworks}

This section briefly exposes some of the most popular argumentation frameworks which extend a basic framework. The frameworks described below add new features to cover concepts, such as preferences or assumptions, used to build more robust and complex argumentation-based systems.

Note that a large variety of frameworks can be found in the literature, but many of them use only one of the frameworks that are presented in this section and are merged with a concrete reasoning technique, such as CBR [Case-based argumentation (Heras et al. 2013)], rule-based reasoning [Rule-based argumentation (Hartfelt et al. 2010)] or knowledge-based reasoning [Information-based argumentation (Sierra and Debenham 2009)]. In summary, this section offers a brief overview of some abstract and general purpose frameworks.

\subsubsection{Preference-based argumentation framework}

To enforce the concept of acceptability, this framework (Amgoud and Cayrol 1998) introduces preference orderings into the definition of acceptability (see Definition 2.5). To express these preferences, a set of new definitions are introduced, as follows:

Definition 2.11 Let $a, b \in A R$,

$a$ defends itself against $b \Longleftrightarrow a$ is preferred to $b$.

Based on Definition 2.11, the concept of defence is modelled by the preference orderings.

Definition 2.12 A preference-based argumentation framework is a triplet $\langle A R, R, P\rangle$, where $P$ is a partial preordering (reflexive and transitive binary relation) on $A R \times A R$.

Because $P$ in this definition of the Preference-based Argumentation Framework (PAF) is a partial relation, the acceptability concept can be weakened. Thus, the acceptability class is defined as follows:

Definition 2.13 Let a preference-based argumentation framework be $\langle A R, R, P\rangle$;

the acceptability class is denoted as $C_{R, \text { Pref }} \subseteq A R$ and is defined as $a \in C_{R, \text { Pref }} \mid \forall b \in$ $A R$ if $(b, a) \in R \Rightarrow(a, b) \in P$.

In other words, Definition 2.13 says that an acceptability class contains all of the arguments that defend themselves against any attack.

Thus, this framework [Preference-based Argumentation Framework (PAF)] can be used if the agents involved in the argumentation process can be modelled with established preferences. Then, they can decide their actions based on their preferences and their environment perceptions. 


\subsubsection{Value-based argumentation framework}

To represent the values to which arguments relate and the ranking of those values, this framework (Bench-Capon 2002), denoted Value-based Argumentation Framework (VAF), extends the definition of Dung's framework (1995).

Definition 2.14 A value-based argumentation framework is a 5-tuple $\langle A R, R, V$, val, valpref $\rangle$, where $A R$ and $R$ are the same as for a standard argumentation framework, $V$ is a non-empty set of values, val is a function on $A R \times V$ that maps arguments to their values, and valpref is a preference relation (transitive, irreflexive and asymmetric) on $V \times V$.

Definition 2.15 Let $a, b \in A R \wedge \operatorname{val}(a), \operatorname{val}(b) \in V, a$ defeats $b \Longleftrightarrow(a, b) \in R \wedge$ $(\operatorname{val}(b), \operatorname{val}(a)) \notin$ valpref.

Furthermore, there is a set of important notions that are defined in Dung's framework (1995) that must be redefined for this framework.

Definition 2.16 Let $a \in A R \wedge S \subseteq A R$; then, $a$ is acceptable with respect to $S \Longleftrightarrow \forall x \in$ $A R \mid x$ defeats $a \Rightarrow \exists y \mid y \in S \wedge y$ defeats $x$.

Definition 2.17 Let $S \subseteq A R ; S$ is conflict-free $\Longleftrightarrow \forall x, y \in S \Rightarrow(x, y) \notin R \vee$ $(\operatorname{val}(y), \operatorname{val}(x)) \in \operatorname{valpref}$.

Summarising, this framework (VAF) can be used if the approach to design the agent behaviour can be defined similar to a measurable reward, i.e., similar to a value of benefit.

\subsubsection{Assumption-based argumentation framework}

This framework (Bondarenko et al. 1993) is focused on how to find arguments, identify attacks and exploit premises that are shared by different arguments. Assumption-based Argumentation Framework (AAF) is defined as follows:

Definition 2.18 An assumption-based argumentation framework is a pair $\langle(L, R), A\rangle$, where $(L, R)$ is a deductive system with a language $L$ and a set of inference rules $R$, and $A \subseteq L$ is a non-empty set of assumptions.

A deductive system enables the formulation of theories, as shown in Definition 2.19.

Definition 2.19 Let any set of formulae $T \subseteq L$ be called a theory. Let $a \in L$, and $T \vdash a$ means that there is a deduction from $T$ whose last element is $a$. A theory $T$ is inconsistent $\Longleftrightarrow T \vdash \perp$, and otherwise, it is consistent. $T h(T)$ is the set $\{a \in L \mid T \vdash a\}$.

The notion of an attack is redefined based on the deductive system included in the framework.

Definition 2.20 Given a theory $T$ and $\Phi, \Delta \subseteq A$ set of assumptions, $\Delta$ attacks $\Phi$ (with respect to $T) \Longleftrightarrow \exists \alpha \neq \perp \wedge \beta \in \Phi \mid T \cup \Delta \vdash \alpha \wedge\{\alpha, \beta\} \vdash \perp$.

The usage of the concept of theory facilitates the internal planning process in an agent because it can formulate some theories and validate them before a negotiation starts. Thus, this framework (AAF) provides a framework that allows reasoning using assumptions, i.e., unknown information that adds uncertainty to the reasoning process. 


\section{Research method}

This work has been performed following the guidelines proposed by Kitchenham and Charters (2007). They propose a formalised process to summarise knowledge in a particular research area for interpreting all of the available research that is related to a specific research question. This process is composed of several steps, which are detailed in the following subsections. Following the Kitchenham and Charters's guidelines (2007), the first step is the definition of a review protocol (Sect. 3.1). The second step is the identification of inclusion and exclusion criteria (Sect. 3.2). The third step is the search for relevant work based on the previously defined criteria (Sect. 3.3). The fourth step is the definition of quality assessments (Sect. 3.4). Finally, the fifth step is the data extraction and synthesis (Sect. 3.5).

\subsection{Review protocol}

We defined the review protocol to specify the methods that were used during the review. The main objectives of this review protocol were to stipulate the background of this systematic review, the research questions, the search processes, the inclusion and exclusion criteria, the selection processes, the quality assessments and the data collection and analysis. The developed review protocol was made by one author and reviewed by the remaining authors to avoid a bias in the review. The background and the research questions are given in previous sections, while the remaining elements are described below.

\subsection{Inclusion and exclusion criteria}

The research studies included in this review must meet the criteria that are established to ensure that all of the relevant studies were found. The time window considered for this review is from 1998 to 2014. Only papers in English from peer-reviewed conferences, workshops and journals were considered for the review. We excluded studies that are not explicitly connected with argumentation techniques for multi-agent systems. Furthermore, informal literature surveys were excluded, such as studies with undefined research questions, search process or data extraction/analysis process.

All of the inclusion criteria must be satisfied to add a study to the review. If any exclusion criterion is met, the study cannot be added. To summarise, Table 1 presents the inclusion and exclusion criteria for this systematic review.

Table 1 Inclusion and exclusion criteria

\author{
Inclusion criteria \\ Studies that are in English \\ Studies that have been published from 1998 to 2014 \\ Studies that focus on argumentation for multi-agent systems \\ Peer-reviewed studies \\ Exclusion criteria \\ Studies that are not related to the research questions \\ Studies with informal literature surveys \\ Studies are not in English \\ Duplicated studies
}




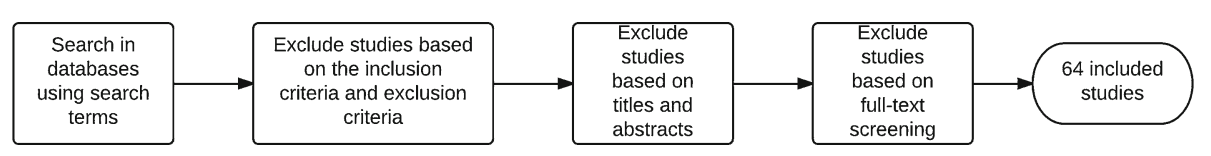

Fig. 1 Stages in the search process

\subsection{Search process}

We have focused on searching the studies in a set of databases composed of the majority of the electronic sources that Brereton et al. (2007) highlight in their work. As Brereton et al. (2007), we accept that the major relevant research results published in books and/or reports are published in scientific papers, also. Nonetheless, if a book presents a high quality description of a specific topic, it can be included in the review.

The electronic sources used in the search process were the following:

- IEEE Xplore (http://www.ieee.org/web/publications/xplore).

- ACM Digital Library (http://dl.acm.org).

- Science Direct (http://www.sciencedirect.com).

- SpringerLink (http://www.springerlink.com).

These databases contain the most important journals and conference proceedings that are relevant for the topic of the review, such as the Autonomous Agents and Multi-agent Systems (AAMAS) Conference or the Argumentation in Multi- Agent Systems (ArgMAS) Workshop.

The concept of argumentation can be used in different ways with similar but not identical meanings; thus, the following search terms were used to find relevant studies in the mentioned databases.

- S1: argumentation multi-agent systems

- S2: argumentation classification

- S3: argumentation negotiation

- S4: argumentation planning

- S5: argumentation dialogue

The selection process to include the relevant studies in the review was conducted by following the steps described below.

1. Search in the databases to find studies by using the search terms.

2. Exclude studies based on the exclusion criteria.

3. Exclude irrelevant studies based on an analysis of their titles and abstracts.

4. Include studies based on a full text read and the inclusion criteria.

The stages of the search process are shown in Fig. 1. Duplicated studies were removed for this review. Three searches were performed at different points in time: July 2012, March 2013 and January 2015, with the intention of covering the publication results in the range of years from 1998 to 2014. During the search processes, a form powered by Google Drive ${ }^{1}$ has been used as an auxiliary tool to extract the relevant data for the studies included in the review, checking the inclusion and exclusion criteria in all of them. Irrelevant publications were removed and, afterward, further filtering was conducted by reading the titles and abstracts. The set of publications that resulted from this step were fully read to ensure that they are relevant to the topic of argumentation techniques for multi-agent systems. The result is a set of 64 studies in the final list.

1 http://drive.google.com. 
Table 2 Data extraction form

\begin{tabular}{|c|c|}
\hline Extracted data & Description \\
\hline Title & Title of the publication \\
\hline Type of study & Book, journal paper, conference paper or workshop paper \\
\hline Source & Database where the study was found \\
\hline Year & Publication year of the study \\
\hline Authors & All of the authors of the publication \\
\hline Affiliation/organisation & The institution of the authors \\
\hline Application field & $\begin{array}{l}\text { The field where the argumentation system is applied, e.g., industrial } \\
\text { management or e-commerce }\end{array}$ \\
\hline Problem type & $\begin{array}{l}\text { The final goal of the argumentation process, e.g., deliberation, } \\
\text { information-seeking or negotiation }\end{array}$ \\
\hline Software environment & $\begin{array}{l}\text { The environment in which the study is developed, e.g., simulation or } \\
\text { argumentation framework }\end{array}$ \\
\hline Support software & Any software tool used to implement the system \\
\hline Agent level & The reasoning technique used by agents, e.g., rules or fuzzy logic \\
\hline Society level & The relations among the agents, e.g., collaborative or competitive \\
\hline Communication protocol & $\begin{array}{l}\text { The protocol used by the agents to communicate among them, e.g., } \\
\text { centralised or FIPA }\end{array}$ \\
\hline Argumentation framework & The name of the argumentation framework used in the study, if any \\
\hline Arguments format & $\begin{array}{l}\text { The format of the shared arguments, e.g., rules, tuples, plain text or logic } \\
\text { formalisms }\end{array}$ \\
\hline Public dataset & If the dataset used in the evaluation is accessible for anyone or not \\
\hline Maturity level & $\begin{array}{l}\text { If the proposed model has been applied in real-life applications or only in } \\
\text { theory }\end{array}$ \\
\hline Real-life data & $\begin{array}{l}\text { If the proposed model has been evaluated with real-life data or only with a } \\
\text { set of examples }\end{array}$ \\
\hline URL & The URL where the study is hosted \\
\hline
\end{tabular}

\subsection{Quality assessment}

The quality criteria established for the review was applied to ensure the quality of each individual study. These criteria are presented below.

1. The study has a description of the research context.

2. The analysis conducted in the study is based on evidence or theoretical reasoning.

3. The evaluation of the research has been performed with real datasets or theoretical explanations.

All of the included studies met each of these three criteria to ensure our confidence in the credibility of any included research.

\subsection{Data extraction and synthesis}

The process of data extraction and synthesis was conducted by reading all of the papers that were included in the review and extracting the relevant data. To maintain consistency in the data extraction process, the form shown in Table 2 has been used to collect interesting data about this particular review. For the data synthesis, the extracted data had been inspected 
Table 3 Citation status of the included studies in detail

\begin{tabular}{lllll}
\hline Cited by & $<5$ & $5-20$ & $20-50$ & $>50$ \\
\hline No. of studies (64) & 27 & 22 & 7 & 8 \\
\hline
\end{tabular}

Table 4 Most cited of the included studies

\begin{tabular}{llr}
\hline Ref. & Title & Citations \\
\hline Sierra et al. (1998) & A framework for argumentation-based negotiation & 340 \\
Amgoud et al. (2000) & Modelling dialogues using argumentation & 301 \\
Kakas and Moraitis (2003) & Argumentation based decision making for autonomous agents & 184 \\
McBurney et al. (2003) & A Dialogue Game Protocol for Agent Purchase Negotiations & 166 \\
Amgoud and Prade (2009) & Using arguments for making and explaining decisions & 155 \\
Amgoud and Parsons (2002) & Agent Dialogues with Conflicting Preferences & 86 \\
Amgoud et al. (2005) & An Argumentation-Based Approach to Multiple Criteria Decision & 86 \\
Vicari et al. (2003) & A multi-agent intelligent environment for medical knowledge & 79 \\
\hline
\end{tabular}

to find similarities, which are used to define how the results could be encapsulated to show them. The results of the synthesis process are described in the following sections.

\section{Overview of the included studies}

This section shows the studies with respect to the publication sources and the citation status to indicate their quality and impact. A set of temporal views are presented in the subsequent sections. All of the selected studies are provided in the references of the paper.

\subsection{Citation status}

Table 3 shows an overview of the citation rates of the studies included in this systematic review. The source of these numbers is Google Scholar. ${ }^{2}$ The aim of these data is not to compare the studies among them. These data are used only to give an indication of the study citation rates. A set of 27 studies have been cited fewer than 5 times. Among these 27 studies, 19 were published between 2012 and 2014; thus, in a short period of time, an increase in their citation numbers is expected in the future. Other 22 studies have been cited between 5 and 20 times, and 7 of them have been cited more than 20 times. Finally, 8 studies have a substantial number of citations and are highlighted in Table 4. Figure 2 presents the status of citations of the included papers by years. This finding also depicts the quality and the impact of these studies. Furthermore, it is expected that these numbers will grow because most of the papers have been published in the last 3 years, as shown in Fig. 3.

\subsection{Temporal view}

Looking at the studies by the year of publication, as shown in Fig. 3, a trend of an increasing number of publications is detected since 2006 for the reviewed topic. The significant increase of publications, especially in the last 3 years, indicates that argumentation techniques are

2 http://scholar.google.es accessed on 23th of February, 2015. 


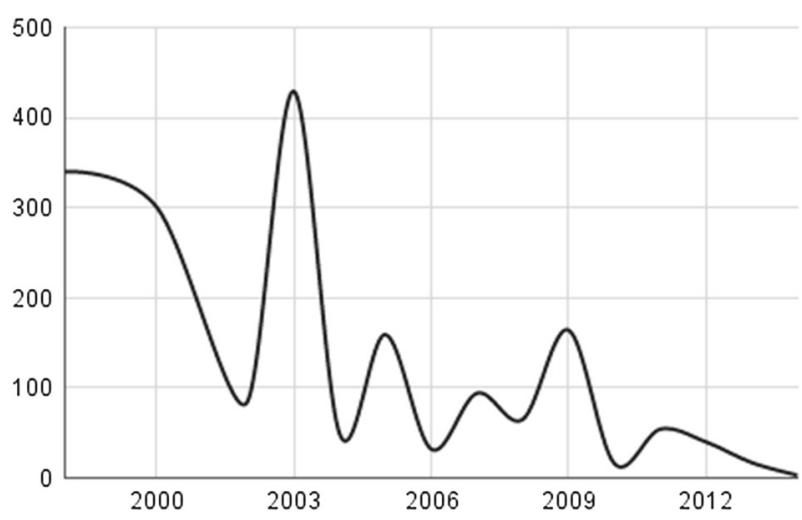

Fig. 2 Status of citations. Axis $\mathrm{X}$ represents year of publication and axis $\mathrm{Y}$ shows number of citations of papers published in that year

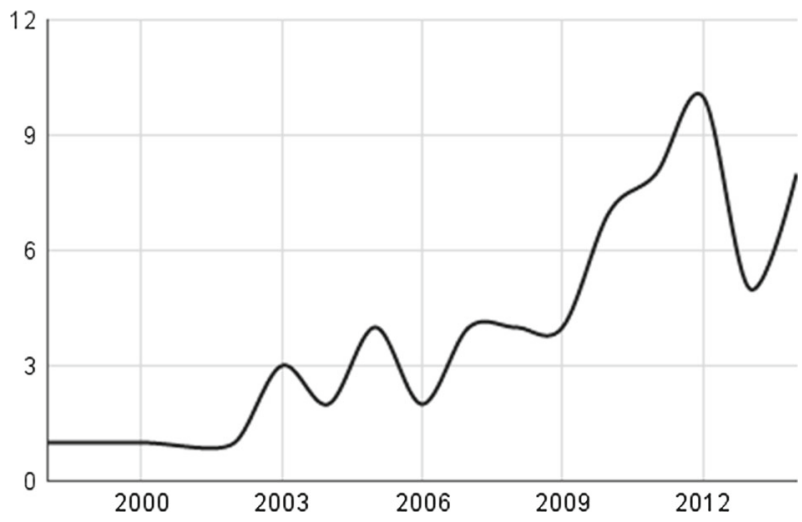

Fig. 3 Quantity of included studies by year

spreading among the community. That increased research interest in the topic reflects that there is still a set of open challenges that can be addressed.

\section{Results}

As described in Sect. 3, during the data synthesis phase, the included studies were compared among them in terms of the research topics and content, to extract knowledge about the use of argumentation techniques. This extraction was performed by using the form presented in Table 2. Among all of the questions presented in that form, we have divided them into two main categories based on two different points of view.

On the one hand, the Application view (Sect. 5.1) shows relevant data about the application of argumentation techniques, such as the application field, the goal of the system proposed in the study or the support software used to implement the system.

On the other hand, MAS view (Sect. 5.2) analyses the design of the proposed approach, highlighting questions such as the reasoning techniques used by the agents, the collaborative or competitive society that they shape, the communication protocol that they use, the argumentation framework that they apply or the format of the arguments that they share. 
Table 5 Basic types of dialogue (Walton and Krabbe 1995)

\begin{tabular}{ll}
\hline Type of dialogue & Goal of dialogue \\
\hline Persuasion & Resolve or clarify issue \\
Inquiry & Prove (disprove) hypothesis \\
Negotiation & Reasonable settlement \\
Information seeking & Exchange information \\
Deliberation & Decide best course of action \\
\hline
\end{tabular}

\subsection{Application view}

This section shows the use of argumentation techniques from the application point of view for the included studies. In other words, the application is analysed by showing the problem type that is resolved, i.e. the goal of the system (Sect. 5.1.1), its application field (Sect. 5.1.2), the support software used in the development of the system (Sect. 5.1.3), the maturity level of the application (Sect. 5.1.4), and finally, its evaluation process (Sect. 5.1.5).

\subsubsection{Problem type}

The application of argumentation techniques in MASs can be used to solve different real-life problems. An argumentation is composed by dialogues among agents and, following the classification made by Walton and Krabbe (1995), there are several basic types of dialogue classified by their goal, as shown in Table 5. Note that these types of dialogue group different tasks. For example, planning and decision making tasks are included in deliberation dialogues, and classification and diagnosis tasks are grouped in inquiry dialogues.

Table 6 shows the included studies classified by the goal of the proposed approach. Some generic studies with a non-specific dialogue type, (Tannai et al. 2011; Gaertner and Toni 2007; Wang and Luo 2010; Caiquan et al. 2010; Hsairi et al. 2006; Hsairi et al. 2010; Obeid and Moubaiddin 2009; Xiong et al. 2012; Gaertner and Toni 2008; Vreeswijk 2005; Amgoud et al. 2000) are not in the table.

\subsubsection{Application field}

The studies that were included in this review are classified in this section based on their application field. First, almost half of them are theoretical studies that have not been applied in real-life applications yet.

The reminder of the included studies presents applications in fields where argumentation is used as a negotiation or classification mechanism, such as e-commerce, to find potentially interesting products (Huang and Lin 2010), to make deals with providers and customers (Ge et al. 2010) or to negotiate supply strategies (Wang et al. 2010). Other interesting application fields are virtual organisations, reasoning with incomplete and conflicting information (Janjua and Hussain 2012), analysing emotional factors (Marreiros et al. 2005), deciding whether or not a person can apply for a specific benefit with a set of restrictions (Wardeh et al. 2012), for credit assignment (Pashaei et al. 2014), to building reputation models (Hsairi et al. 2010), conflict resolution in supply chains (Hsairi et al. 2006) and building ambient intelligent systems (Moraitis and Spanoudakis 2007). In industrial management, these techniques are applied in management systems for multiple tasks, such as to decide the way to dry an oven in automobile production (Ye et al. 
Table 6 Studies per dialogue type

\begin{tabular}{|c|c|c|}
\hline Type & No. studies & Studies \\
\hline Persuasion & 4 & $\begin{array}{l}\text { van der Weide et al. (2011), Heras et al. (2013), Heras et al. (2013), } \\
\text { Amgoud and Parsons (2002) }\end{array}$ \\
\hline Inquiry & 9 & $\begin{array}{l}\text { Maio et al. (2011), Amgoud and Serrurier (2007), Keppens (2011), } \\
\text { Rowe et al. (2012), Wardeh et al. (2012), Amgoud and Serrurier } \\
\text { (2008), Tao et al. (2014), Fogli et al. (2013), Vicari et al. (2003) }\end{array}$ \\
\hline Negotiation & 17 & $\begin{array}{l}\text { Yuan et al. (2009), Ye et al. (2010), Ge et al. (2010), Wang et al. } \\
\text { (2010), Bulling et al. (2008), Monteserin and Amandi (2011), El-Sisi } \\
\text { and Mousa (2012), Brandao Neto et al. (2013), Xue-jie et al. (2013), } \\
\text { Maio and Silva (2014), Pashaei et al. (2014), Sierra et al. (1998), } \\
\text { Amgoud et al. (2008), McBurney et al. (2003), Amgoud (2006), } \\
\text { Morge and Beaune (2004), Alonso (2004) }\end{array}$ \\
\hline Information seeking & 2 & Huang and Lin (2010), Bedi and Vashisth (2014) \\
\hline Deliberation & 21 & $\begin{array}{l}\text { Moraitis and Spanoudakis (2007), Liu et al. (2010), Yuan et al. (2011), } \\
\text { Das (2005), Aulinas et al. (2012), Janjua and Hussain (2012), Zhang } \\
\text { et al. (2012), Chow et al. (2013), Velaga et al. (2012), Grando et al. } \\
\text { (2012), Harvey et al. (2007), Wang et al. (2014), Kakas and Moraitis } \\
\text { (2003), Fan et al. (2014), d'Avila Garcez et al. (2014), Thomopoulos } \\
\text { et al. (2015), Ferrando and Onaindia (2013), Fan et al. (2013), } \\
\text { Amgoud and Prade (2009), Amgoud et al. (2005), Tang and Parsons } \\
\text { (2005) }\end{array}$ \\
\hline
\end{tabular}

2010), to manage waste-water discharges (Aulinas et al. 2012), to decide about an automatic freight process (Chow et al. 2013) or to improve the performance of transport systems in rural areas (Velaga et al. 2012). Knowledge engineering is another interesting field in which some studies apply argumentation techniques for different tasks, such as to make automatic matching among existing ontologies or knowledge bases (Maio et al. 2011; Maio and Silva 2014) or to discuss about a particular issue in medical guidelines (Grando et al. 2012). For some critical tasks, argumentation techniques are applied too, such as an emergency rescue (Zhang et al. 2012) or security administration (Rowe et al. 2012).

Finally, argumentation techniques have been applied in many different domains, what shows their suitability for complex and heterogeneous environments.

\subsubsection{Support software}

This section describes a summary of the support software that is used in the included studies. At this point, we must remark that not all of the studies offer information about which software, platforms or frameworks are used to implement the work.

Among the included studies, one of the most common approaches (Moraitis and Spanoudakis 2007; Wang et al. 2010; Xiong et al. 2012; El-Sisi and Mousa 2012) is to use generic purpose multi-agent platforms, such as JADE, to implement an argumentation framework required by the problem under consideration. However, other studies use some tools that are focused on the argumentation framework, such as CASAPI (Gaertner and Toni 2007) or PISA (Wardeh et al. 2012). Furthermore, some platforms, such as Magentix 2 agent platform (Heras et al. 2013), mix several approaches and can be used to develop argumentation in multi-agent systems. Finally, other studies (Janjua and Hussain 2012; Marreiros et al. 2005) implement argumentation mechanisms using web applications. 
Table 7 Maturity level

\begin{tabular}{|c|c|c|}
\hline Maturity level & No. studies & Studies \\
\hline Theory & 35 & $\begin{array}{l}\text { Yuan et al. (2009), Liu et al. (2010), Wang and Luo (2010), Caiquan } \\
\text { et al. (2010), Ge et al. (2010), Hsairi et al. (2006), Hsairi et al. (2010), } \\
\text { Obeid and Moubaiddin (2009), Yuan et al. (2011), Maio et al. (2011), } \\
\text { Das (2005), van der Weide et al. (2011), Bulling et al. (2008), } \\
\text { Amgoud and Serrurier (2007), Keppens (2011), Rowe et al. (2012), } \\
\text { Gaertner and Toni (2008), Vreeswijk (2005), Amgoud and Serrurier } \\
\text { (2008), Brandao Neto et al. (2013), Tao et al. (2014), Amgoud et al. } \\
\text { (2000), Kakas and Moraitis (2003), d'Avila Garcez et al. (2014), } \\
\text { Sierra et al. (1998), Fan et al. (2013), Amgoud and Parsons (2002), } \\
\text { Amgoud et al. (2008), Amgoud and Prade (2009), McBurney et al. } \\
\text { (2003), Amgoud et al. (2005), Amgoud (2006), Tang and Parsons } \\
\text { (2005), Morge and Beaune (2004), Alonso (2004) }\end{array}$ \\
\hline Prototype & 22 & $\begin{array}{l}\text { Moraitis and Spanoudakis (2007), Ye et al. (2010), Wang et al. (2010), } \\
\text { Monteserin and Amandi (2011), Heras et al. (2013), Huang and Lin } \\
\text { (2010), Janjua and Hussain (2012), Xiong et al. (2012), Zhang et al. } \\
\text { (2012), El-Sisi and Mousa (2012), Heras et al. (2013), Grando et al. } \\
\text { (2012), Harvey et al. (2007), Wardeh et al. (2012), Xue-jie et al. } \\
\text { (2013), Wang et al. (2014), Fogli et al. (2013), Fan et al. (2014), Bedi } \\
\text { and Vashisth (2014), Ferrando and Onaindia (2013), Maio and Silva } \\
\text { (2014), Pashaei et al. (2014) }\end{array}$ \\
\hline Application & 7 & $\begin{array}{l}\text { Tannai et al. (2011), Gaertner and Toni (2007), Aulinas et al. (2012), } \\
\text { Chow et al. (2013), Velaga et al. (2012), Thomopoulos et al. (2015), } \\
\text { Vicari et al. (2003) }\end{array}$ \\
\hline
\end{tabular}

\subsubsection{Maturity level}

This section describes the application of the model proposed in the studies for real-life applications based on their maturity level. We have categorised all of the included studies at three different levels. The Theory level represents a proof of concept that is made in a lab but not developed or applied for any real-life application. All of the studies included in the Prototype level describe the results of initial work applied to real-life problems. Finally, Application level studies give an overview for a system used in a real-life task. The studies are classified using these criteria in Table 7.

Furthermore, in the temporal view of the studies classified per maturity level shown in Table 8, we found an increase of the number of studies that includes prototypes and applications instead of only theoretical explanations, which means argumentation techniques are being applied to software systems in recent years.

\subsubsection{Evaluation process of the proposed model}

The last two questions that are included in this section of the review are whether the study results have been validated with real data and whether the proposed models have been compared with other similar and alternative methods or models.

The first of these aspects used to analyse the studies is the usage of real data to evaluate the proposed models. At this point, we distinguish three different levels (see Table 9): "No, only theory" for studies that use only some simple data to probe the proposed model in a specific case; "Yes, real but private" for studies that evaluate their models with real data but the experiments are not replicable because their data are private or not available for anyone; 
Table 8 Maturity level of included studies per year

\begin{tabular}{lccl}
\hline Year & Theory & Prototype & Application \\
\hline $1998-2005$ & 11 & 0 & 1 \\
$2006-2010$ & 15 & 5 & 1 \\
$2011-2014$ & 9 & 17 & 5
\end{tabular}

Table 9 Studies per evaluation with real datasets

\begin{tabular}{|c|c|c|}
\hline Real datasets & No. studies & Studies \\
\hline No, only theory & 50 & $\begin{array}{l}\text { Yuan et al. (2009), Moraitis and Spanoudakis (2007), Gaertner and } \\
\text { Toni (2007), Liu et al. (2010), Wang and Luo (2010), Caiquan } \\
\text { et al. (2010), Ge et al. (2010), Hsairi et al. (2006), Hsairi et al. } \\
\text { (2010), Obeid and Moubaiddin (2009), Wang et al. (2010), Yuan } \\
\text { et al. (2011), Maio et al. (2011), van der Weide et al. (2011), } \\
\text { Bulling et al. (2008), Amgoud and Serrurier (2007), Keppens } \\
\text { (2011), Monteserin and Amandi (2011), Heras et al. (2013), } \\
\text { Huang and Lin (2010), Xiong et al. (2012), Zhang et al. (2012), } \\
\text { El-Sisi and Mousa (2012), Gaertner and Toni (2008), Harvey } \\
\text { et al. (2007), Vreeswijk (2005), Amgoud and Serrurier (2008), } \\
\text { Brandao Neto et al. (2013), Tao et al. (2014), Xue-jie et al. } \\
\text { (2013), Wang et al. (2014), Amgoud et al. (2000), Kakas and } \\
\text { Moraitis (2003), Fogli et al. (2013), Fan et al. (2014), Bedi and } \\
\text { Vashisth (2014), d'Avila Garcez et al. (2014), Thomopoulos et al. } \\
\text { (2015), Ferrando and Onaindia (2013), Pashaei et al. (2014), } \\
\text { Sierra et al. (1998), Amgoud and Parsons (2002), Amgoud et al. } \\
\text { (2008), Amgoud and Prade (2009), McBurney et al. (2003), } \\
\text { Amgoud et al. (2005), Amgoud (2006), Tang and Parsons (2005), } \\
\text { Morge and Beaune (2004), Alonso (2004) }\end{array}$ \\
\hline Yes, real but private & 12 & $\begin{array}{l}\text { Tannai et al. (2011), Ye et al. (2010), Das (2005), Aulinas et al. } \\
\text { (2012), Janjua and Hussain (2012), Rowe et al. (2012), Chow } \\
\text { et al. (2013), Heras et al. (2013), Velaga et al. (2012), Grando } \\
\text { et al. (2012), Vicari et al. (2003), Fan et al. (2013) }\end{array}$ \\
\hline Yes, public data & 2 & Wardeh et al. (2012), Maio and Silva (2014) \\
\hline
\end{tabular}

and finally, "Yes, public data" for studies that offer a public repository of datasets to allow the replicability of the experiments.

The second aspect to classify a study in this section is whether the proposed model is compared with other alternative models or techniques (see Table 10).

\subsection{MAS view}

Once the studies included in this review have been analysed from the point of view of their applications, the design of the MAS is figured out in this section based on the following criteria. Section 5.2.1 presents the reasoning techniques used by the agents. Section 5.2.2 shows the behaviour that agents have in their society. Section 5.2.3 analyses the environment in which the MAS is executed. Section 5.2.4 exposes which communications protocols are used in the system. Section 5.2.5 offers an overview of what argumentation framework used in the included studies. Finally, Sect. 5.2.6 shows the format that is used to interchange arguments among agents. 
Table 10 Studies per evaluation with comparison

\begin{tabular}{lll}
\hline Comparison & No. studies & Studies \\
\hline \multirow{2}{*}{57} & Tannai et al. (2011), Yuan et al. (2009), Moraitis and Spanoudakis \\
& (2007), Gaertner and Toni (2007), Liu et al. (2010), Wang and Luo \\
& (2010), Ye et al. (2010), Caiquan et al. (2010), Ge et al. (2010), Hsairi \\
& et al. (2006), Hsairi et al. (2010), Obeid and Moubaiddin (2009), Wang \\
& et al. (2010), Yuan et al. (2011), Maio et al. (2011), Das (2005), van der \\
& Weide et al. (2011), Bulling et al. (2008), Amgoud and Serrurier \\
& (2007), Keppens (2011), Monteserin and Amandi (2011), Huang and \\
& Lin (2010), Aulinas et al. (2012), Janjua and Hussain (2012), Xiong \\
& et al. (2012), Zhang et al. (2012), Rowe et al. (2012), Chow et al. \\
& (2013), Velaga et al. (2012), Grando et al. (2012), Gaertner and Toni \\
& (2008), Harvey et al. (2007), Vreeswijk (2005), Amgoud and Serrurier \\
& (2008), Brandao Neto et al. (2013), Tao et al. (2014), Xue-jie et al. \\
& (2013), Wang et al. (2014), Amgoud et al. (2000), Kakas and Moraitis \\
& (2003), Fogli et al. (2013), Fan et al. (2014), Bedi and Vashisth (2014), \\
& d'Avila Garcez et al. (2014), Thomopoulos et al. (2015), Vicari et al. \\
& (2003), Sierra et al. (1998), Fan et al. (2013), Amgoud and Parsons \\
& (2002), Amgoud et al. (2008), Amgoud and Prade (2009), McBurney \\
& et al. (2003), Amgoud et al. (2005), Amgoud (2006), Tang and Parsons \\
& (2005), Morge and Beaune (2004), Alonso (2004) \\
Heras et al. (2013), El-Sisi and Mousa (2012), Heras et al. (2013), & Wardeh et al. (2012), Ferrando and Onaindia (2013), Maio and Silva \\
& (2014), Pashaei et al. (2014) \\
&
\end{tabular}

\subsubsection{Agent level}

This section exposes the techniques that were used by agents internally to process their data, such as the evidences or arguments, in other words, how an agent reasons internally to decide its behaviours and reactions.

Based on the included studies in the review, the most commonly used technique is rulebased inference (Hsairi et al. 2006; Gaertner and Toni 2007; Moraitis and Spanoudakis 2007; Yuan et al. 2009; Obeid and Moubaiddin 2009; Caiquan et al. 2010; Tannai et al. 2011; Xiong et al. 2012; Harvey et al. 2007; Huang and Lin 2010; Janjua and Hussain 2012; Wardeh et al. 2012; Rowe et al. 2012; van der Weide et al. 2011). However, there are other techniques that must not be ignored because they offer other alternatives to reason under uncertainty, such as fuzzy logic (Liu et al. 2010; Hsairi et al. 2010; Chow et al. 2013; Wang and Luo 2010; Tao et al. 2014; Bedi and Vashisth 2014) or Bayesian inference (Vreeswijk 2005; Das 2005; Keppens 2011; Vicari et al. 2003), to reason based on similarity, such as CBR (Heras et al. 2013; Aulinas et al. 2012; Heras et al. 2013), or to measure the benefit of a specific action using utility functions (Ge et al. 2010).

It must be mentioned that many theoretical studies that are included in the review do not offer any information about any reasoning technique for the agent level, because they are focused on the argumentation task, not on the reasoning process itself.

\subsubsection{Society level}

As the interaction among agents in a multi-agent system can be collaborative or competitive depending on the goals of each agent, the behaviour at the society level is studied in this section. 
Table 11 Studies per agent level behaviour

\begin{tabular}{lll}
\hline Behaviour & No. studies & Studies \\
\hline Collaborative & 38 & Hsairi et al. (2006), Moraitis and Spanoudakis (2007), Yuan et al. \\
& (2009), Obeid and Moubaiddin (2009), Liu et al. (2010), Ge et al. \\
& (2010), Hsairi et al. (2010), Ye et al. (2010), Maio et al. (2011), Zhang \\
& et al. (2012), El-Sisi and Mousa (2012), Aulinas et al. (2012), Chow \\
& et al. (2013), Grando et al. (2012), Harvey et al. (2007), Marreiros et al. \\
& (2005), Rowe et al. (2012), Velaga et al. (2012), Letia and Groza \\
& (2012), Amgoud and Serrurier (2008), Monteserin and Amandi (2011), \\
& Wang et al. (2010), Das (2005), Bulling et al. (2008), Amgoud and \\
& Serrurier (2007), Huang and Lin (2010), Janjua and Hussain (2012), \\
& Tao et al. (2014), Wang et al. (2014), Kakas and Moraitis (2003), Fogli \\
& et al. (2013), Fan et al. (2014), Bedi and Vashisth (2014), Ferrando and \\
& Onaindia (2013), Maio and Silva (2014), Vicari et al. (2003), Tang and \\
& Parsons (2005), Morge and Beaune (2004) \\
& Yuan et al. (2011), Keppens (2011), Heras et al. (2013), Heras et al. \\
& (2013), Brandao Neto et al. (2013), Xue-jie et al. (2013), Pashaei et al. \\
& (2014), Sierra et al. (1998), Amgoud and Parsons (2002), Gaertner and \\
& Toni (2007), Caiquan et al. (2010), Tannai et al. (2011), Xiong et al. \\
& (2012), Wardeh et al. (2012), van der Weide et al. (2011), Vreeswijk \\
(2005)
\end{tabular}

Table 12 Studies per execution environment

\begin{tabular}{lll}
\hline Environment & No. studies & Studies \\
\hline Application & 8 & $\begin{array}{c}\text { Zhang et al. (2012), Rowe et al. (2012), Chow et al. (2013), Grando } \\
\text { et al. (2012), Fogli et al. (2013), Bedi and Vashisth (2014), Vicari } \\
\text { et al. (2003), Huang and Lin (2010) }\end{array}$ \\
& 7 & $\begin{array}{l}\text { Ye et al. (2010), Wang et al. (2010), Ge et al. (2010), Wang et al. } \\
\text { (2014), Heras et al. (2013), Heras et al. (2013), Pashaei et al. (2014) }\end{array}$
\end{tabular}

At this point, we identify two different behaviours: collaborative, a set of agents have a common goal and collaborate to achieve it; or competitive, every agent has its own goal and competes to earn the maximum possible benefit. Table 11 shows this aspect for the included studies. Some general frameworks or approaches are not included in this classification because they support both behaviours depending on the environment.

\subsubsection{Execution environment}

Another interesting aspect to consider is the environment where the agents are executed. We classify two different main types of execution environment for the included studies, as shown in Table 12. The type which is most directly related to the final systems is the application environment, i.e., the multi-agent system presented in the study shows a system in which the MAS is integrated into a real system. The simulation type presents a study where the complexity of the scenario is too high to develop a real application for a probe of concept; as a result, it can be considered to be a previous stage of the application environment. Finally, the theoretical studies are not included in Table 12. 
Table 13 Studies per communication protocol

\begin{tabular}{|c|c|c|}
\hline Comm. Protocol & No. studies & Studies \\
\hline FIPA & 10 & $\begin{array}{l}\text { Moraitis and Spanoudakis (2007), Obeid and Moubaiddin (2009), } \\
\text { Wang et al. (2010), Monteserin and Amandi (2011), Huang and Lin } \\
\text { (2010), Xiong et al. (2012), El-Sisi and Mousa (2012), Ge et al. } \\
\text { (2010), Fan et al. (2014), Vicari et al. (2003) }\end{array}$ \\
\hline Centralised & 8 & $\begin{array}{l}\text { Rowe et al. (2012), Wang et al. (2014), Kakas and Moraitis (2003), } \\
\text { Fogli et al. (2013), Tannai et al. (2011), Keppens (2011), Wardeh } \\
\text { et al. (2012), Thomopoulos et al. (2015) }\end{array}$ \\
\hline
\end{tabular}

\subsubsection{Communication protocol}

In any multi-agent system, the communication among agents plays an important role in the complexity of the final system. In industrial applications, communication protocols, such as the Foundation for Intelligent Physical Agents (FIPA) protocol (O'Brien and Nicol 1998; Specification 2000) or the Knowledge Query and Manipulation Language (KQML) protocol (Finin et al. 1994), are the best practice (Ferber et al. 2004). However, in prototypes of theoretical explanations, other ad hoc alternatives are widely used too.

Table 13 shows two different categories which have been used to analyse all of the included studies. As shown in Table 13, FIPA protocol is quite common in studies which are in an advanced development stage, i.e. developed prototype or application. But, a centralised approach were all agents executed in the same node is quite common too. Note that some of the studies do not offer any information about how information is interchanged among the agents. Thus, those studies are not considered in this section.

\subsubsection{Argumentation framework}

In the literature, we can find argumentation frameworks with approaches that deal with different aspects to achieve a given goal. Table 14 shows an overview of the argumentation frameworks that are used explicitly in the studies include in this review. Studies which do not identify a specific argumentation framework are not included in this section. For example, if an argumentative approach applied in a study is using some of the concepts presented by Dung (1995) or Amgoud and Cayrol (1998), but authors do not make any reference their works, that study will not be included in the corresponding row in Table 14.

Furthermore, a discussion of the frameworks in Table 14 is included in Sect. 6.2.4 analysing their features and applications from a practical point of view.

\subsubsection{Argument format}

Among the existing alternatives to exchange information during an argumentation process, the non-theoretical studies included in the review use the formats shown in Table 15.

The most popular option between the included studies is the use of rules that explain the arguments, facilitating its understanding. Conforming the arguments as individuals of an ontology offers some powerful benefits, as the checking of the arguments coherence or the use of external ontologies to add expressiveness to the argumentation dialogue. Moreover, the FIPA-ACL standard support the use of ontologies during the agent conversation. Nevertheless, 
Table 14 Studies per argument framework

\begin{tabular}{|c|c|c|}
\hline Argument format & No. studies & Studies \\
\hline DAF (Dung 1995) & 11 & $\begin{array}{l}\text { Tannai et al. (2011), Caiquan et al. (2010), Yuan et al. (2011), } \\
\text { van der Weide et al. (2011), Amgoud and Serrurier (2007), } \\
\text { Huang and Lin (2010), Xiong et al. (2012), Rowe et al. } \\
\text { (2012), Vreeswijk (2005), Wang et al. (2014), Bedi and } \\
\text { Vashisth (2014) }\end{array}$ \\
\hline $\begin{array}{l}\text { PAF (Amgoud and Cayrol } \\
\text { 1998) }\end{array}$ & 5 & $\begin{array}{l}\text { Obeid and Moubaiddin (2009), Bulling et al. (2008), Amgoud } \\
\text { and Serrurier (2008), Amgoud et al. (2005), Amgoud (2006) }\end{array}$ \\
\hline VAF (Bench-Capon 2002) & 4 & $\begin{array}{l}\text { Heras et al. (2013), Heras et al. (2013), d'Avila Garcez et al. } \\
\text { (2014), Thomopoulos et al. (2015) }\end{array}$ \\
\hline $\begin{array}{l}\text { AAF (Bondarenko et al. } \\
\text { 1993) }\end{array}$ & 4 & $\begin{array}{l}\text { Gaertner and Toni (2007), Gaertner and Toni (2008), Fan et al. } \\
\text { (2014), Fan et al. (2013) }\end{array}$ \\
\hline ABN (Rahwan et al. 2003) & 11 & $\begin{array}{l}\text { Ye et al. (2010), Hsairi et al. (2006), Zhang et al. (2012), El-Sis } \\
\text { and Mousa (2012), Harvey et al. (2007), Brandao Neto et al. } \\
\text { (2013), Xue-jie et al. (2013), Sierra et al. (1998), Amgoud } \\
\text { et al. (2008), Morge and Beaune (2004), Alonso (2004) }\end{array}$ \\
\hline $\begin{array}{l}\text { TLAF (Maio and Silva } \\
\text { 2012) }\end{array}$ & 2 & Maio et al. (2011), Maio and Silva (2014) \\
\hline LPwNF (Kakas et al. 1994) & 2 & Moraitis and Spanoudakis (2007), Kakas and Moraitis (2003) \\
\hline
\end{tabular}

Table 15 Studies per argument format

\begin{tabular}{lll}
\hline $\begin{array}{l}\text { Argument format } \\
\text { FIPA-ACL }\end{array}$ & No. studies & Studies \\
Ontologies & 3 & $\begin{array}{l}\text { Ge et al. (2010), Wang et al. (2010), El-Sisi and Mousa (2012) } \\
\text { Huang and Lin (2010), Janjua and Hussain (2012), Xiong et al. (2012), } \\
\text { Heras et al. (2013) }\end{array}$ \\
Plain text & 4 & $\begin{array}{c}\text { Liu et al. (2010), Rowe et al. (2012), Fogli et al. (2013), Vicari et al. } \\
(2003)\end{array}$ \\
Rules & 7 & $\begin{array}{c}\text { Tannai et al. (2011), Moraitis and Spanoudakis (2007), Monteserin and } \\
\text { Amandi (2011), Chow et al. (2013), Vreeswijk (2005), Wardeh et al. } \\
\text { (2012), Tao et al. (2014) }\end{array}$ \\
& &
\end{tabular}

some studies uses arguments in plain text format which offers a great flexibility to express any idea and facilitate the human interaction with the argumentation system.

Note that some studies do not offer information about the format for interchange in argument. In other words, they do not refer to how arguments are formatted or use a generic tuple to represent them. Thus, these studies are not included in this section, because they express arguments in a theoretical way.

Table 16 shows the use of these argument formats in the included studies during the temporal window of this review. The developed prototypes in recent years, shown in Sect. 5.1.4, have motivated an increase of the number of studies that specify a format to interchange arguments, such as ontologies or rules.

\section{Discussion}

The identified categories shown in Sect. 5 provide an overview of the argumentation techniques for multi-agent systems research as well as a basis for discovering possibilities for the improvement of research and practice. 
Table 16 Argument formats count per years for included studies

\begin{tabular}{lllll}
\hline Year & FIPA-ACL & Ontologies & Plain text & Rules \\
\hline $1998-2005$ & 0 & 0 & 1 & 1 \\
$2006-2010$ & 0 & 1 & 1 & 1 \\
$2011-2014$ & 3 & 3 & 2 & 5 \\
\hline
\end{tabular}

The following sections discuss the scope of the review (Sect. 6.1) and the potential impact on research and practice (Sect. 6.2) as well as the validity threats to this review (Sect. 6.3).

\subsection{Scope of the systematic review}

This paper focuses on the development of methods that are based on argumentation techniques and the application of those methods in multi-agent systems. Thus, it includes only studies that address pragmatic aspects, such as the development or definition of innovative techniques and systems that provide useful solutions to real-life problems. This systematic review focuses mainly on the studies that describe approaches that are concerned with argumentation methods applied to multi-agent systems. Nevertheless, theoretical studies that can be potentially applied in real-life systems are included to address the problem.

Within the area of non-monotonic reasoning, the more general concept of Defeasible Reasoning was introduced by Pollock (1987). The use of this type of reasoning has led to useful and important findings in other research areas, such as intelligent agents and MAS (Dix et al. 2009). Using dialectical approaches, agents can share arguments to claim a conclusion or counter-arguments to reject invalid statements. The use of argumentation techniques can handle these situations with well formalised dialogue models that can be applied in MASs.

Within the area of multi-agent systems, one of the most important challenges and recommendations (Winikoff 2012) is to focus on the "macro" level, i.e., to develop techniques for designing and implementing interactions and to integrate micro (single cognitive agent) and macro (MAS) design and implementation. At macro level, argumentation can be used to define agents interactions and environment policies. While, at micro level, agents generates and evaluates arguments based on their own reasoning processing. Thus, argumentation techniques integrate both macro and micro level, as Winikoff recommends in Winikoff (2012).

Thus, as can be seen from the above, there are many possibilities in the application of the argumentation techniques for the evolution of MASs. This review is focused on analysis of the recent work in that field, to obtain valuable conclusions for further research.

\subsection{Impact on research and practice}

This systematic review has a number of implications for research and practice. The following sections expose the maturation of the technology to apply argumentation techniques in MASs for real-life problems (Sect. 6.2.1), the theoretical foundation of the studies (Sect. 6.2.2), the combination of approaches to address complex systems (Sect. 6.2.3) and, finally, some guidelines to apply argumentation these techniques (Sect. 6.2.4).

\subsubsection{Technology maturation}

This section discussion where the argumentation technology for multi-agent systems stands today. Redwine Jr and Riddle (1985) identify one initial phase and five different stages, which 
are briefly commented below, for technology maturation to broadly spread the use of any new software technology.

The initial phase is called Basic Research and constitutes general recognition of problem and discussion of its scope and nature. The first stage is Concept Formulation, which is the convergence on a compatible set of ideas. The second stage, Development and Extension, is the clarification of the underlying ideas and the extension of the general approach to a broader solution. The third stage, called Internal Enhancement and Exploration, is a major extension of general approach to other problem domains, an use of the technology to solve real problems, stabilisation and porting of the technology. The fourth stage is External Enhancement and Exploration, which is the same activities as for the previous stage but carried out by a broader group, including people outside the development group. Finally, the fifth stage, Popularisation, is the appearance of production-quality, supported versions commercialisation and marketing of the technology, propagation of the technology throughout community of users.

The argumentation technology can be considered to be in the third stage, because it has a robust and solid theoretical background as an interdisciplinary field study and is being applied in some applications domains, as shown in Sect. 5.1 and its subsections. Given the steady increase of the studies that present results from a prototype in recent years, as shown in Table 8, it is possible to state that solutions which apply this technology to real-life problems will be available in the future. This implies that is early to place argumentation technology in fourth stage, because there is a small number of MAS platforms that support argumentation techniques. The appearance of more platforms of this type would foster the development and application of this technology, promoting its adoption for a broader audience.

\subsubsection{Theoretical foundation and formalisation for argumentation techniques}

In previous sections, the included studies have been classified in several ways. One of them has been the maturity level of the proposed approach (see Sect. 5.1.4). Among the 64 studies that were included in this review, approximately one-half $(54.68 \%)$ of them are theoretical studies that formalise or prove advanced concepts about the use of argumentation theory in MAS. Approximately one-third (34.37\%) of them present prototypes of a system that attempts to resolve real-life problems. Finally, only one-tenth $(10.93 \%)$ of them expose a final application of this technology.

Thus, based on these data and on the previous studies that were not included in the scope of this review due to time constraints (only studies published between 1998 and 2014 are included), we can suggest the argumentation techniques have a wide theoretical foundation and are in an early third stage of the Redwine and Riddle classification (1985).

\subsubsection{Combining approaches to address a multifaceted perspective of multi-agent systems and argumentation techniques}

The use of argumentation techniques to handle different ambiguous situations in a MAS can be a correct decision in many cases. However, these techniques by themselves do not solve all of the issues that are found typically in a MAS when they have to face real-life problems. For example, how agents decide to expose an argument to the group or how they choose an argument to attempt a counter-attack are issues that are not always covered in theoretical studies, even when they are keys issues when a MAS is used in a real-life application, and some agents must interact with external systems that have incomplete or irrelevant information. For this reason, some studies include the use of argumentation with other reasoning techniques, 
such as case-based reasoning [Case-based argumentation (Heras et al. 2013)], rule-based reasoning [Rule-based argumentation (Hartfelt et al. 2010)] or knowledge-based reasoning [Information-based argumentation (Sierra and Debenham 2009)].

Furthermore, another interesting approach is the use of ontologies to represent the arguments (see Sect. 5.2.6). It allows the agents to perform semantic reasoning with the information that is available in the complete ontology and, even, to make matching of different ontologies (Maio and Silva 2014). Following this approach, some argument formats have been proposed, such as the Argument Interchange Format (AIF) (Chesñevar et al. 2006) or ArgDF (Zablith 2007).

\subsubsection{Tailoring relevant approaches for specific contexts}

This section provides some guidelines to apply argumentation techniques in a MAS for reallife applications. We identify three different steps to consider in each specific context: (i) analyse the suitability of these techniques, (ii) select an argumentation framework, and (iii) choose a MAS platform.

First step is evaluating the suitability of argumentation techniques for the considered problem. As we have reviewed in Sects. 5.1.1 and 5.1.2, argumentation techniques have already been applied in MAS for a number of problems and domains, respectively. Authors can review those sections to analyse designs for similar problems or domains. As argumentation techniques define mechanisms for carrying out complex and sophisticated dialogues, they are suitable for designing agents that interact among them offering and processing reasons, i.e. rational agents. In particular, argumentation techniques can be applied for achieving different types of goals in agent interactions, such as resolve or clarify an issue, prove an hypothesis, get reasonable settlements, decide future actions or simply exchange information among agents (Walton and Krabbe 1995).

Second step is selecting the argumentation framework. Based on the data gathered in Sects. 5.2.5 and 5.1.2, we provide some guidelines for this selection below.

The most popular general purpose argumentation framework is the Dung's Ar- gumentation Framework (DAF) (Dung 1995), that defines basic concepts for any argumentation process. It presents the notion of acceptability based on an attack relation between two arguments and proposes a set of interesting semantics for reasoning. However, DAF presents some lacks not considering the strength of an attack, what is addressed in the Value-based Argumentation Framework (VAF) (Bench-Capon 2002), or the existence of preferences between arguments, that is proposed in the Preference-based Argumentation Framework (PAF) (Amgoud and Cayrol 1998). Thus, PAF provides an adequate solution when preference orderings between arguments is interesting to get better solution of the considered problem. For example, in collaborative problem solving, PAF could be applied if a solution is better (i.e. preferred) than other (Obeid and Moubaiddin 2009). If those preferences can be expressed as a numeric value to measure their strengths, VAF presents an interesting solution, as those values can be learnt dynamically from data (d'Avila Garcez et al. 2014) or from agents experience (Heras et al. 2013).

Moreover, a variant of DAF that adds the notion of assumptions and preferences is found in the Assumption-based Argumentation Framework (AAF) (Bondarenko et al. 1993). Particularly, AAF presents an appropriate approach if arguments are expressed as deductions based on a set of assumptions (Dung et al. 2009).

As negotiation cannot be understood without argumentation (Dung 1995), Argumentationbased Negotiation (ABN) (Sierra et al. 1998; Rahwan et al. 2003) framework explores the possibility that negotiating agents aim at satisfying their own individuals goals, and optionally 
can have shared goals. This framework is able to combine cooperative and competitive contexts to achieve efficient solutions (Xue-jie et al. 2013).

For changing environments, Logic Programming without Negation as Failure (LPwNF) (Kakas et al. 1994) approach, extended in Kakas and Moraitis (2003), allows a high degree of flexibility in the adaptation of the argumentative reasoning of agents (Moraitis and Spanoudakis 2007) based on their social contexts, i.e. the same argument can be accepted or rejected according to the relation between agents. This framework is interesting to contexts in which relations among roles of agents change the acceptability of an argument. For example, in a military hierarchy, a soldier agent accepts or discusses arguments generated by a captain or a soldier, respectively.

Finally, if no one of the previous frameworks covers the context of problem under consideration, Three-Layer Argumentation Framework (TLAF) (Maio and Silva 2012) can be an interesting option. The proposed three-layers model is composed by a Meta-Model Layer, an Instance Layer which both combined present the classical relations between arguments that can be found in other frameworks, such as DAF or VAF, and a Model Layer which aim to capture semantics of the specific application domain (e.g. e-commerce or legal reasoning). This Model Layer should be adapted for the specific domain under consideration. This framework has been successfully applied in an ontology matching domain (Maio and Silva 2014; Maio et al. 2011).

Third step is selecting a MAS platform that supports the argumentation framework. As reviewed in Sect. 5.1.3, there is not yet a widely accepted argumentative platform for MAS. Thus, in case the problem requires, researchers should extend the selected MAS framework to support the needed argumentation facilities.

\subsection{Validity threats}

There are two main threats to validity in this systematic review. The first threat is bias in our selection of the included studies, and the second threat is the data extraction and synthesis.

To identify relevant studies and ensure that the process of selection has been unbiased, a research method was developed (see Sect. 3) to define research questions, inclusion and exclusion criteria, and the search process. The review protocol was defined by the first author, and then it was reviewed by the remaining authors, to check the formulation of the research questions, the validity of the search strings and the extracted data.

To ensure correctness in the data extraction and synthesis process, a form was defined using Google Drive ${ }^{3}$ to obtain consistent data to answer the research questions and analyse the data by using the facilities that offer the online support tool.

\section{Conclusions}

This paper presents a systematic review of argumentation techniques for multi-agent system research. This review has been conducted following the principles provided by Kitchenham and Charters in Kitchenham and Charters (2007).

The search process has been previously defined, and three searches were performed in July 2012, March 2013 and January 2015. Based on inclusion and exclusion criteria, a set of 64 studies were included in this review and were properly analysed and compared. The extraction and synthesis process was previously established to answer the most relevant aspects of the

3 http://drive.google.com. 
included studies from the authors point of view. The results are clearly presented in the sections of this paper, both graphically and literally.

While a quantitative overview is presented in Sect. 5, a qualitative discussion is included in Sect. 6. The findings from both sections are summarised below as follows:

Regarding the goal of the dialogue, the most common objective of an argumentative system is to decide the best course of action, i.e. decision making or planning. Other common goal is to achieve reasonable settlement or agreement among the agents through a negotiation process. However, the goal of inquiry is quite common in tasks such as diagnosis or classification. For further information, see Sect. 5.1.1.

The included studies address many different applications fields, but we must highlight the use of argumentation techniques in e-commerce and virtual organisations to make arrangements among the parts that are interested in a specific problem or in industrial management, to monitor some constraints or to improve the performance of the processes. For further reading, see Sect. 5.1.2.

Regarding the MAS platforms included in the studies, a number of them extend general purpose platforms, such as JADE, with the required argumentation capabilities for the problem under consideration. Other researchers, in contrast, tend to use their own specific frameworks, such as CASAPI or PISA, as shown in Sect. 5.1.3.

Another interesting finding is the evolution of the studies from pure theoretical studies to prototypes or real-life applications, as analysed in Sect. 6.2.1. The number of prototypes/applications described in the period from 2011 to 2014 depicts this technology offers approaches suitable for real-life applications.

Most researchers tend to use rule systems for reasoning with arguments. Other alternatives are CBR, fuzzy logic or Bayesian reasoning, as shown in Sect. 5.2.1. Furthermore, the use of rules as interchange format is the most common alternative to share arguments. However, other alternatives have been applied in non-theoretical approaches, such as arguments as individuals of an ontology or plain text arguments, as shown in Sect. 5.2.6.

Paying attention to the agents' interactions in their society, Sect. 5.2.2 highlights that a collaborative behaviour is observed more often than a competitive behaviour. Nevertheless, it is clear that argumentation techniques have been applied successfully in both cooperative and competitive environments.

Based on the number of studies that present developed prototypes and real-life applications in recent years, we can place the argumentation technology for multi-agent systems in an early third stage of the maturation classification proposed by Redwine Jr and Riddle (1985). As an interdisciplinary field of study, argumentation has a robust and solid theoretical background and it is beginning to be applied in industrial applications, as shown in Sect. 6.2.1.

Some guidelines to apply argumentation techniques in a specific context are offered in Sect. 6.2.4. Moreover, that section includes a discussion about the features of the most popular argumentation frameworks from a practical point of view.

To conclude, the findings expose that the usage of argumentation techniques are mainly used in academia researchers, and non-broadly applied to real-life applications yet, i.e. the available technology begins to offer some non-mature solutions for industrial applications. The lack of a standard format to share arguments limits the proliferation of tools and testbeds. But, some efforts have been done in that direction, such as the Argument Interchange Format (AIF) (Chesñevar et al. 2006) or ArgDF (Zablith 2007). Furthermore, the appearance of more MAS platforms that support argumentation techniques (such as Magentix2) ${ }^{4}$ would foster its adoption for a broader audience. However, the increased interest in the development

4 http://www.gti-ia.upv.es/sma/tools/magentix2/. 
of prototypes, shown in Sect. 5.1.4, suggests this technology is getting closer to their broader application to real-life environments.

Acknowledgments This research work is supported by the Spanish Ministry of Economy and Competitiveness under the R\&D project CALISTA (TEC2012-32457); by the Spanish Ministry of Industry, Energy and Tourism under the R\&D project BigMarket (TSI-100102-2013-80); and, by the Autonomous Region of Madrid through the program MOSI-AGIL-CM (Grant P2013/ICE-3019, co-funded by EU Structural Funds FSE and FEDER). The authors want to acknowledge the useful suggestions of the reviewers which have increased significantly the quality of the review.

Open Access This article is distributed under the terms of the Creative Commons Attribution 4.0 International License (http://creativecommons.org/licenses/by/4.0/), which permits unrestricted use, distribution, and reproduction in any medium, provided you give appropriate credit to the original author(s) and the source, provide a link to the Creative Commons license, and indicate if changes were made.

\section{References}

Alonso E (2004) Rights and argumentation in open multi-agent systems. Artif Intell Rev 21(1):3-24

Amgoud L (2006) An argumentation-based model for reasoning about coalition structures. In: Parsons S, Maudet N, Moraitis P, Rahwan I (eds) Argumentation in multi-agent systems, vol 4049., Lecture notes in computer scienceSpringer, Berlin, pp 217-228

Amgoud L, Bonnefon JF, Prade H (2005) An argumentation-based approach to multiple criteria decision. In: Godo L (ed) Symbolic and quantitative approaches to reasoning with uncertainty, vol 3571., Lecture notes in computer scienceSpringer, Berlin, pp 269-280

Amgoud L, Cayrol C (1998) On the acceptability of arguments in preference-based argumentation. In: Proceedings of the 14th conference on incertainty in artificial intelligence. UAI'98Morgan Kaufmann Publishers Inc., San Francisco, CA, USA, pp 1-7

Amgoud L, Dimopoulos Y, Moraitis P (2008) A general framework for argumentation-based negotiation. In: Rahwan I, Parsons S, Reed C (eds) Argumentation in multi-agent systems, vol 4946., Lecture notes in computer scienceSpringer, Berlin, pp 1-17

Amgoud L, Maudet N, Parsons S (2000) Modelling dialogues using argumentation. In: Proceedings of the 4th international conference on multiagent systems, 2000, pp 31-38

Amgoud L, Parsons S (2002) Agent dialogues with conflicting preferences. In: Meyer JJ, Tambe M (eds) Intelligent agents VIII, vol 2333., Lecture notes in computer scienceSpringer, Berlin, pp 190-205

Amgoud L, Prade H (2009) Using arguments for making and explaining decisions. Artif Intell 173(34):413436

Amgoud L, Serrurier M (2007) Arguing and explaining classifications. In: Proceedings of the 6th international joint conference on autonomous agents and multiagent systems, AAMAS '07. ACM, New York, NY, USA, pp 160:1-160:7

Amgoud L, Serrurier M (2008) Agents that argue and explain classifications. Auton Agent Multi Agent Syst 16(2):187-209

Aulinas M, Tolchinsky P, Turon C, Poch M, Cort U (2012) Argumentation-based framework for industrial wastewater discharges management. Eng Appl Artif Intell 25(2):317-325

Bedi P, Vashisth P (2014) Empowering recommender systems using trust and argumentation. Inf Sci 279:569586

Bench-Capon TJ, Dunne PE (2007) Argumentation in artificial intelligence. Artif Intell 171(10):619-641

Bench-Capon TJM (2002) Value based argumentation frameworks. CoRR cs.AI/0207059

Bondarenko A, Toni F, Kowalski RA (1993) An assumption-based framework for non-monotonic reasoning. Proceedings of the 2nd international workshop on logic programming and non-monotonic reasoning. MIT Press, New York, pp 171-189

Brandao Neto P, Rocha A, Lopes Cardoso H (2013) Risk assessment through argumentation over contractual data. In: 2013 8th Iberian conference on information systems and technologies (CISTI), pp 1-6

Brereton P, Kitchenham B, Budgen D, Turner M, Khalil M (2007) Lessons from applying the systematic literature review process within the software engineering domain. J Syst Softw 80(4):571-583

Bulling N, Dix J, Chesñevar CI (2008) Modelling coalitions: Atl + argumentation. In: Proceedings of the 7th international joint conference on autonomous agents and multiagent systems, vol 2, AAMAS '08, pp 681-688 International Foundation for Autonomous Agents and Multiagent Systems 
Caiquan X, Chunzhi W, Qing M, Xianbin S (2010) An argumentation-based interaction model and its algorithms in multi-agent system. In: International conference on intelligent computation technology and automation (ICICTA), 2010, vol 1, pp 493-496

Chesñevar C, McGinnis J, Modgil S, Rahwan I, Reed C, Simari G, South M, Vreeswijk G, Willmott S (2006) Towards an argument interchange format. Knowl Eng Rev 21(4):293-316

Chow HK, Siu W, Chan CK, Chan HC (2013) An argumentation-oriented multi-agent system for automating the freight planning process. Expert Syst Appl 40(10):3858-3871

d'Avila Garcez AS, Gabbay DM, Lamb LC (2014) A neural cognitive model of argumentation with application to legal inference and decision making. J Appl Logic 12(2):109-127

Das S (2005) Symbolic argumentation for decision making under uncertainty. In: 2005 8th International conference on information fusion, vol 2, p 8

Dix J, Parsons S, Prakken H, Simari G (2009) Research challenges for argumentation. Comput Sci Res Dev 23(1):27-34

Dung PM (1995) On the acceptability of arguments and its fundamental role in nonmonotonic reasoning, logic programming and n-person games. Artif Intell 77(2):321-357

Dung PM, Kowalski RA, Toni F (2009) Assumption-based argumentation. Argumentation in artificial intelligence. Springer, New York, pp 199-218

El-Sisi A, Mousa H (2012) Argumentation based negotiation in multiagent system. In: 7th International conference on computer engineering systems (ICCES), 2012, pp 261-266

Fan X, Craven R, Singer R, Toni F, Williams M (2013) Assumption-based argumentation for decision-making with preferences: a medical case study. In: Leite J, Son T, Torroni P, van der Torre L, Woltran S (eds) Computational logic in multi-agent systems, vol 8143., Lecture notes in computer scienceSpringer, Berlin, pp 374-390

Fan X, Toni F, Mocanu A, Williams M (2014) Dialogical two-agent decision making with assumption-based argumentation. In: Proceedings of the 2014 international conference on autonomous agents and multiagent systems. AAMAS 14 international foundation for autonomous agents and multiagent systems, Richland, SC, pp 533-540

Ferber J, Gutknecht O, Michel F (2004) From agents to organizations: an organizational view of multi-agent systems. Agent-oriented software engineering IV. Springer, New York, pp 214-230

Ferrando SP, Onaindia E (2013) Context-aware multi-agent planning in intelligent environments. Inf Sci 227:22-42

Feteris ET (1999) Fundamentals of legal argumentation: a survey of theories on the justification of judicial decisions, vol 1. Boom Koninklijke Uitgevers

Finin T, Fritzson R, McKay D, McEntire R (1994) Kqml as an agent communication language. In: Proceedings of the 3rd international conference on information and knowledge management, pp 456-463

Fogli D, Giacomin M, Stocco F, Vivenzi F (2013) Supporting medical discussions through an argumentationbased tool. In: Proceedings of the biannual conference of the Italian chapter of SIGCHI, CH Italy '13. ACM, New York, NY, USA, pp 18:1-18:10

Gaertner D, Toni F (2007) Computing arguments and attacks in assumption-based argumentation. IEEE Intell Syst 22(6):24-33

Gaertner D, Toni F (2008) Preferences and assumption-based argumentation for conflict-free normative agents. In: Rahwan I, Parsons S, Reed C (eds) Argumentation in multi-agent systems, vol 4946., Lecture notes in computer scienceSpringer, Berlin, pp 94-113

Ge Z, Guo-rui J, Ti-yun H (2010) Design of argumentation-based multi-agent negotiation system oriented to e-commerce. Int Conf Internet Technol Appl 2010:1-6

Grando MA, Glasspool D, Boxwala A (2012) Argumentation logic for the flexible enactment of goal-based medical guidelines. J Biomed Inform 45(5):938-949

Hartfelt A, Järvinen AS, Vila MAS (2010) Rule-based argumentation. Master's thesis, ITU

Harvey P, Chang C, Ghose A (2007) Support-based distributed search: a new approach for multiagent constraint processing. Argumentation in multi-agent systems, vol 4766. Lecture notes in computer science, Springer, Berlin, pp 91-106

Heras S, Jordán J, Botti V, Julián V (2013) Argue to agree: a case-based argumentation approach. Int J Approx Reason 54(1):82-108

Heras S, Jordn J, Botti V, Julin V (2013) Case-based strategies for argumentation dialogues in agent societies. Inf Sci 223:1-30

Hsairi L, Ghedira K, Alimi A, Benabdelhafid A (2010) Trust and reputation model for r2-ibn framework. IEEE Int Conf Syst Man Cybern 2010:2137-2144

Hsairi L, Ghedira K, Alimi M, Benabdelhafid A (2006) Resolution of conlicts via argument based netotiation: extended enterprise case. In: International conference on service systems and service management, 2006, vol 1, pp 828-833 
Huang SL, Lin CY (2010) The search for potentially interesting products in an e-marketplace: an agent-to-agent argumentation approach. Expert Syst Appl 37(6):4468-4478

Janjua NK, Hussain FK (2012) Webidss-argumentation-enabled web-based idss for reasoning over incomplete and conflicting information. Knowl Based Syst 32:9-27

Kakas A, Moraitis P (2003) Argumentation based decision making for autonomous agents. In: Proceedings of the 2nd international joint conference on autonomous agents and multiagent systems. AAMAS '03, ACM, New York, NY, USA, pp 883-890

Kakas AC, Mancarella P, Dung PM (1994) The acceptability semantics for logic programs. ICLP 94:504-519

Keppens J (2011) On extracting arguments from bayesian network representations of evidential reasoning. In: Proceedings of the 13th international conference on artificial intelligence and law. ICAIL '11ACM, New York, NY, USA, pp 141-150

Kitchenham B, Charters S (2007) Guidelines for performing systematic literature reviews in software engineering. Technical report EBSE 2007-001, Keele University and Durham University Joint Report

Letia I, Groza A (2012) Justifying argument and explanation in labelled argumentation. IEEE Int Conf Intell Comput Commun Process 2012:11-18

Liu X, Wanchoo R, Arvapally R (2010) Intelligent computational argumentation for evaluating performance scores in multi-criteria decision making. Int Symp Collab Technol Syst 2010:143-152

Maio P, Silva N (2012) A three-layer argumentation framework. Theorie and applications of formal argumentation. Springer, New York, pp 163-180

Maio P, Silva N (2014) An extensible argument-based ontology matching negotiation approach. Science of computer programming, 95, Part 1(0), 3-25. Special Issue on Systems Development by Means of Semantic Technologies

Maio P, Silva N, Cardoso J (2011) Generating arguments for ontology matching. In: 22nd international workshop on database and expert systems applications (DEXA), 2011, pp 239-243

Marreiros G, Ramos C, Neves J (2005) Dealing with emotional factors in agent based ubiquitous group decision. In: Lecture notes in computer science, pp 41-50

Maudet N, Parsons S, Rahwan I (2007) Argumentation in multi-agent systems: context and recent developments. Argumentation in multi-agent systems. Springer, New York, pp 1-16

McBurney P, Van Eijk R, Parsons S, Amgoud L (2003) A dialogue game protocol for agent purchase negotiations. Auton Agent Multi Agent Syst 7(3):235-273

Monteserin A, Amandi A (2011) Argumentation-based negotiation planning for autonomous agents. Decis Support Syst 51(3):532-548

Moor Ad, Aakhus M (2006) Argumentation support: from technologies to tools. Commun ACM 49(3):93-98

Moraitis P, Spanoudakis N (2007) Argumentation-based agent interaction in an ambient-intelligence context. IEEE Intell Syst 22(6):84-93

Morge M, Beaune P (2004) A negotiation support system based on a multi-agent system: specificity and preference relations on arguments. Proceedings of the 2004 ACM symposium on applied computing, SAC '04. ACM, New York, pp 474-478

Obeid N, Moubaiddin A (2009) On the role of dialogue and argumentation in collaborative problem solving. In: 9th International conference on intelligent systems design and applications (ISDA), 2009, pp 1202-1208

O'Brien PD, Nicol RC (1998) Fipatowards a standard for software agents. BT Technol J 16(3):51-59

Pashaei K, Taghiyareh F, Badie K (2014) A negotiation-based genetic framework for multi-agent credit assignment. In: Mller J, Weyrich M, Bazzan A (eds) Multiagent system technologies, vol 8732., Lecture notes in computer scienceSpringer, New York, pp 72-89

Perelman C, Olbrechts-Tyteca L (1969) The new rhetoric: a treatise on argumentation. University of Notre Dame Press, Notre Dame

Pollock JL (1987) Defeasible reasoning. Cogn Sci 11(4):481-518

Rahwan I, Ramchurn SD, Jennings NR, McBurney P, Parsons S, Sonenberg L (2003) Argumentation-based negotiation. Knowl Eng Rev 18:343-375

Redwine ST Jr, Riddle WE (1985) Software technology maturation. In: Proceedings of the 8th international conference on software engineering. ICSE '85IEEE Computer Society Press, Los Alamitos, CA, USA, pp 189-200

Rowe J, Levitt K, Parsons S, Sklar E, Applebaum A, Jalal S (2012) Argumentation logic to assist in security administration. In: Proceedings of the 2012 workshop on New security paradigms. NSPW ' $12 \mathrm{ACM}$, New York, NY, USA, pp 43-52

Sierra C, Debenham J (2009) Information-based argumentation. Knowledge representation for agents and multi-agent systems. Springer, New York, pp 130-144

Sierra C, Jennings N, Noriega P, Parsons S (1998) A framework for argumentation-based negotiation. In: Singh M, Rao A, Wooldridge M (eds) Intelligent agents IV agent theories, architectures, and languages, vol 1365., Lecture notes in computer scienceSpringer, Berlin, pp 177-192 
Specification FICA (2000) Foundation for intelligent physical agents

Tang Y, Parsons S (2005) Argumentation-based dialogues for deliberation. Proceedings of the 4th international joint conference on autonomous agents and multiagent systems, AAMAS '05. ACM, New York, pp 552559

Tannai S, Goto Y, Maruyama Y, Itoya T, Hagiwara T, Sawamura H (2011) A versatile argumentation system based on the logic of multiple-valued argumentation. In: 11th International conference on hybrid intelligent systems (HIS), pp 370-376

Tao X, Miao Y, Zhang Y, Shen Z (2014) Collaborative medical diagnosis through fuzzy petri net based agent argumentation. In: IEEE international conference on fuzzy systems (FUZZ-IEEE), pp 1197-1204

Thomopoulos R, Croitoru M, Tamani N (2015) Decision support for agri-food chains: a reverse engineering argumentation-based approach. Ecol Inf 26: 182-191

Toulmin SE (2003) The uses of argument. Cambridge University Press, Cambridge

van der Weide TL, Dignum F, Meyer JJC, Prakken H, Vreeswijk GAW (2011) Multi-criteria argument selection in persuasion dialogues. The 10th international conference on autonomous agents and multiagent systems vol 3, AAMAS '11International Foundation for Autonomous Agents and Multiagent Systems. Richland, SC, pp 921-928

van Eemeren FH, Grootendorst RF, Henkemans FS (1996) Fundamentals of argumentation theory: a handbook of historical backgrounds and contemporary applications

Velaga NR, Rotstein ND, Oren N, Nelson JD, Norman TJ, Wright S (2012) Development of an integrated flexible transport systems platform for rural areas using argumentation theory. Res Trans Bus Manage 3:62-70

Vicari RM, Flores CD, Silvestre AM, Seixas LJ, Ladeira M, Coelho H (2003) A multi-agent intelligent environment for medical knowledge. Artif Intell Med 27(3):335-366 Software Agents in Health Care

Vreeswijk G (2005) Argumentation in bayesian belief networks. Argumentation in multi-agent systems, vol 3366. Lecture notes in computer science. Springer, Berlin, pp 111-129

Wallace KR (1963) The substance of rhetoric: good reasons. Q J Speech 49(3):239-249

Walton D (2009) Argumentation theory: a very short introduction. In: Simari G, Rahwan I (eds) Argumentation in artificial intelligence. Springer, New York, pp 1-22

Walton DN, Krabbe ECW (1995) Commitment in dialogue: basic concepts of interpersonal reasoning. State University of New York Press, New York

Wang B, Luo G (2010) Extend argumentation frameworks based on degree of attack. In: 2010 9th IEEE international conference on cognitive informatics (ICCI), pp 771-776

Wang G, Wong TN, Wang X (2010) A negotiation protocol to support agent argumentation and ontology interoperability in mas-based virtual enterprises. In: 7th International conference on information technology: new generations (ITNG), pp 448-453

Wang Y, Yao L, Liu B, Xu J (2014) An argumentation based feedback system for action planning of service robots. In: 2014 International conference on multisensor fusion and information integration for intelligent systems (MFI), pp 1-7

Wardeh M, Coenen F, Bench-Capon T (2012) Multi-agent based classification using argumentation from experience. Auton Agent Multi Agent Syst 25(3):447-474

Winikoff M (2012) Challenges and directions for engineering multi-agent systems. CoRR, abs/1209.1428

Xiong C, Xiang W, Ouyang Y (2012) Argumentation in multi-agent system based on jade. In: 3rd International conference on intelligent control and information processing (ICICIP), pp 88-91

Xue-jie D, Jian C, Ying-lan H, Guo-rui J, Ti-yun H (2013) Multi-attribute negotiation model based on internal factors argumentation. In: 2013 International conference on management science and engineering (ICMSE), pp 20-27

Ye Y, Lin H, Chen G, Liu Z (2010) Argumentation-based negotiation in multi-agent system of drying oven for automobile body. In: IEEE international conference on intelligent computing and intelligent systems (ICIS), vol 2, pp 453-456

Yuan J, Bao A, Yao L, Qi X, Liu F (2009) Defeasible logic base bdi agent for argumentation. IEEE international conference on intelligent computing and intelligent systems 1:223-228

Yuan J, Yao L, Hao Z, Liu F, Yuan T (2011) Multi-party dialogue games for distributed argumentation system. In: Proceedings of the 2011 IEEE/WIC/ACM international conferences on web intelligence and intelligent agent technology, vol 02. WI-IAT '11IEEE Computer Society, Washington, DC, USA, pp $329-332$

Zablith F (2007) Argdf: Arguments on the semantic web. Master's thesis, The British University in Dubai Jointly with The University of Edinburgh

Zhang W, Liang Y, Ji S, Tian Q (2012) Argumentation agent based fire emergency rescue project making. IEEE Symp Robot Appl 2012:892-895 